\title{
Modulation of the Ribonucleotide Reductase-Antimetabolite Drug Interaction in Cancer Cell Lines
}

\author{
Jun Zhou, ${ }^{1,2}$ Paula Oliveira, ${ }^{1,2}$ Xueli Li, ${ }^{1}$ Zhengming Chen, ${ }^{1,2}$ and Gerold Bepler, ${ }^{1,2}$ \\ ${ }^{1}$ Department of Thoracic Oncology, Moffitt Cancer Center, Tampa, FL 33612, USA \\ ${ }^{2}$ Department of Oncology, Karmanos Cancer Institute, Detroit, MI 48201, USA \\ Correspondence should be addressed to Gerold Bepler, beplerg@karmanos.org
}

Received 29 March 2010; Revised 14 July 2010; Accepted 1 September 2010

Academic Editor: Caroline Kisker

Copyright ( $) 2010$ Jun Zhou et al. This is an open access article distributed under the Creative Commons Attribution License, which permits unrestricted use, distribution, and reproduction in any medium, provided the original work is properly cited.

\begin{abstract}
RRM1 is a determinant of gemcitabine efficacy in cancer patients. However, the precision of predicting tumor response based on RRM1 levels is not optimal. We used gene-specific overexpression and RNA interference to assess RRM1's impact on different classes of cytotoxic agents, on drug-drug interactions, and the modulating impact of other molecular and cellular parameters. RRM1 was the dominant determinant of gemcitabine efficacy in various cancer cell lines. RRM1 also impacted the efficacy of other antimetabolite agents. It did not disrupt the interaction of two cytotoxic agents when combined. Cell lines with truncation, deletion, and null status of p53 were resistant to gemcitabine without apparent relationship to RRM1 levels. Pemetrexed and carboplatin sensitivity did not appear to be related to p53 mutation status. The impact of p53 mutations in patients treated with gemcitabine should be studied in prospective clinical trials to develop a model with improved precision of predicting drug efficacy.
\end{abstract}

\section{Introduction}

The regulatory subunit of ribonucleotide reductase (RRM1) has been identified as the key molecular determinant of gemcitabine efficacy both in vitro and in vivo [1-7]. Human lung and pancreatic cancer cell lines and a serially transplanted mouse colon cancer made resistant to gemcitabine through continuous exposure to increasing amounts of drug overexpressed RRM1 [1,3,5]. RRM1 overexpression through transfection of a lung cancer cell line likewise resulted in gemcitabine resistance [4]. Reduction of RRM1 expression through RNA interference abrogated the induced gemcitabine resistance and increased drug sensitivity in otherwise sensitive cell lines $[4,5]$.

An association between intratumoral RRM1 levels and efficacy of systemic therapy that includes gemcitabine as a single-agent or in combination with a platinumagent or pemetrexed has also been reported [8]. However, the addition of a vinca-alkaloid (vinorelbine) to a gemcitabine-containing combination in patients with nonsmallcell lung cancer (NSCLC) appeared to abrogate the RRM1-gemcitabine efficacy association [2]. Although gemcitabine therapy is statistically significantly more efficacious in patients with low tumoral RRM1 levels, the scatter plots reported and correlation coefficients are less than optimal for precise predictions on whether or not gemcitabine will result in tumor shrinkage in individual patients [7].

Here we studied associations between RRM1 expression levels and sensitivities to frequently used chemotherapeutic single agents and combinations as well as cell lines characteristics in an effort to determine the impact of RRM1 on relevant classes of agents and to identify parameters that might modify the RRM1-gemcitabine efficacy interaction.

\section{Material and Methods}

2.1. Cell Lines and Culture Conditions. The cell lines used in this study were obtained from the American Type Culture Collection (ATCC) or the originators. MCF7 human mammary adenocarcinoma cells were maintained in MEM- $\alpha$ supplemented with $10 \%$ fetal bovine serum, penicillin/streptomycin, nonessential aminoacids $(0.1 \mathrm{mM})$, sodium pyruvate $(1 \mathrm{mM})$, sodium bicarbonate $(1.5 \mathrm{~g} / \mathrm{L})$, and bovine pancreatic insulin (Sigma Aldrich, $0.01 \mathrm{mg} / \mathrm{mL}$ ). All NSCLC cell lines and HCT8 (human colonic adenocarcinoma cells) were maintained in RPMI 1640 supplemented 
with L-glutamine $(2 \mathrm{mM})$, penicillin/streptomycin (100 units $/ 100 \mu \mathrm{g}$ per $\mathrm{mL}$ ), and $10 \%$ fetal bovine serum. Unless otherwise specified, all reagents were purchased from Gibco (Invitrogen). All cell lines were free of mycoplasma contamination (Stratagene), their authenticity was confirmed by DNA fingerprint analysis, and testing was performed within 6 months of in vitro propagation for experiments described herein. They were harvested at $70 \%$ confluency for subsequent experiments.

2.2. RRM1 and $p 53$ Transfected Cell Lines. We have generated three human cell line models derived from lung (H23), breast (MCF7), and colon (HCT8) cancers, with increased and decreased RRM1 expression by stable transfection as previously described [9]. In general, stably overexpressing RRM1 cell lines and their controls were generated by transfection with full-length human RRM1 cDNA cloned into the expression plasmid pCMV-Tag2 (Stratagene). Stably downregulated RRM1 cell lines were generated by transfection with pSUPER-GFP (oligoEngine) containing RRM1-specific target sequence (GACGCTAGAGCGGTCTTAT) or, as a control, scramble sequence that had no similarity to any known gene using FuGENE HD (Roche Applied Science). The overexpression and down regulation of RRM1 were confirmed by real-time RT-PCR and immunoblotting. A stably TP53 wild-type expressing cell line (H358-p53+) was generated by transfection with a pcDNA3 vector containing full-length TP53 cDNA (a gift from Dr. Jiandong Chen).

2.3. Target Gene Expression Reduction. Dharmacon onTARGETplus Smartpool siRNA to TP53, ERCC1, and RRM1 (Dharmacon RNAi Technologies) were delivered to H23, A549, H292, and H460 NSCLC cell lines using Lipofectamine RNAiMAX (Invitrogen) following manufacturer's instructions. Nontarget Pool siRNA was used as control.

\subsection{Isolation of Total Cellular RNA and Real-Time PCR.} Total RNA was isolated from cultured cells with TRIzol reagent (Invitrogen), and cDNA was synthesized with the Superscript amplification kit (Invitrogen). Quantitative realtime PCR was employed to measure the expression of RRM1 using 18s-rRNA as internal reference standard. The RRM1 primers were forward AAGAGCAGCGTGCCA GAGAT, reverse ACACA TCAAA GACCA GTCCT GATTA G, and probe $5^{\prime}$ TTTGC TCTTT GGATT CCGGA TCTCT TCA $3{ }^{\prime}$. 18s-rRNA was detected using commercial primers and probes (Applied Biosystems). For each sample, the target RRM1 and 18s-rRNA concentrations were determined by interpolation to a standard curve. The normalized RRM1 quantity was then derived by dividing the RRM1 value by the 18s-rRNA value.

2.5. Drug Sensitivity and In Vitro Proliferation Assay. The following anticancer drugs were tested: gemcitabine and pemetrexed (Eli Lilly), methotrexate, carboplatin, hydroxyurea, and 5-fluorouracil (Sigma Aldrich); docetaxel (SanofiAventis); cisplatin (Ben Venue Laboratory), vinorelbine (Sicor), and etoposide (Bedford Laboratory). At the time of use, the drugs were freshly prepared and diluted stepwise to the desired concentration in the proper solvent or culture medium.

Cell viability in response to various drugs was assessed with a cell proliferation 3-(4,5-dimethylthiazol-2-yl)-5-(3carboxymethoxyphenyl)-2-(4-sulfophenyl)-2H-tetrazolium (MTS) assay in 96-well plates (Corning). Briefly, 1,0004,000 viable cells were seeded in triplicate in $100 \mu \mathrm{L}$ of growth medium and allowed to attach for $24 \mathrm{~h}$. The cells were then continuously exposed to $0.01 \mathrm{nM}-1 \mathrm{mM}$ of each drug (0.1 nM-10 mM for hydroxyurea) for 3-10 days. Thereafter, cells were exposed to CellTiter96 AQueous One Solution Reagent (Promega) for $2 \mathrm{~h}$ at $37^{\circ} \mathrm{C}$, and formazan absorbance was measured at $490 \mathrm{~nm}$ using a microplate reader (Benchmark Plus, Bio-Rad). Each experiment was repeated 3 times on different days with separate preparations of cells and drugs.

Alternately, drug activity was assessed using the CellTiterBlue viability assay in 384-well plates (Promega). In this format, 800 or 1,200 cells (for 5-day or 3-day experiments, respectively) were plated in each well by using a Precision $\mathrm{XS}$ automated pipetting system (Bio-Tek Instruments) and allowed to attach overnight at $37^{\circ} \mathrm{C}$. The respective drugs and combinations were serially diluted in growth medium, and $5 \mu \mathrm{l}$ were then added to wells. Four replicate wells were used for each drug concentration and an additional four control wells received media without drug. After 3 or 5 days of incubation, $5 \mu \mathrm{l}$ CellTiter-Blue solution was added to each well. Cell viability was assessed by the ability of the metabolically active cells to reduce resazurin to the highly fluorescent resorufin. The resulting fluorescence $(560 \mathrm{Ex} / 590 \mathrm{Em})$ was measured with a Synergy HT microplate reader (Bio-Tek Instruments).

For both, the 96-well and 384-well experiments, fluorescence data were transferred to a spreadsheet program to calculate the percent viability relative to the replicate control cell wells that did not receive drug. Data analysis for $\mathrm{IC}_{50}$ value calculations was performed using SigmaPlot (Systat Software).

For drug combination experiments, the IC50 values obtained from single drug assays were used to design the experiments, and the cell viability assays were performed as described above. The results were analyzed for synergistic, additive, or antagonistic effects using the combination index (CI) method developed by Chou [10]. For the application of this method, the drug concentration dilutions were used at fixed dose ratios (e.g., 50:1, 2:5, 1:250). Briefly, the doseeffect curve for each drug alone was determined based on experimental observations using the median-effect principle and compared to the effect achieved with a combination of two drugs to derive a CI value. The method involves plotting dose-effect curves, for each agent and their combination, using the median-effect equation: $\mathrm{fa} / \mathrm{fu}=(D / \mathrm{Dm}) m$, where $D$ is the dose of the drug, $\mathrm{Dm}$ the dose required for a $50 \%$ effect (equivalent to IC50), fa and fu the affected and unaffected fractions ( $\mathrm{fa}=1-\mathrm{fu}$ ), and $m$ the exponent signifying the sigmoidicity of the dose-effect curve. The computer software XLfit was used to calculate the values of Dm and $m$. The CI used for the analysis of the drug combinations 
was determined by the isobologram equation for mutually nonexclusive drugs that have different modes of action: $\mathrm{CI}=(D)_{1} /(D x)_{1}+(D)_{2} /(D x)_{2}+(D)_{1}(D)_{2} /(D x)_{1}(D x)_{2}$, where $(D x)_{1}$ and $(D x)_{2}$ in the denominators are the doses (or concentrations) for drug 1 and drug 2 alone that gives $\mathrm{x} \%$ inhibition, whereas $(D)_{1}$ and $(D)_{2}$ in the numerators are the doses of drug 1 and drug 2 in combination that also inhibited $x \%$ (i.e., isoeffective). Combination indices $\mathrm{CI}<1$, $\mathrm{CI}=1$, and $\mathrm{CI}>1$ indicate synergism, additive effects, and antagonism, respectively.

2.6. Immunoblotting and Antibody Reagents. Tumor cells were cultured as described above. Crude cell extract proteins were suspended in RIPA buffer in the presence of a protease inhibitor cocktail. After determination of the protein concentration, extracts were separated on $8 \%-10 \%$ SDS-PAGE gels, transferred to membranes, and the expression profiles analyzed by immunoblotting. Monoclonal antibodies or antisera to RRM1 (T-16, cat \# sc-11733, lot \# H0608), RRM2a (I15, cat \# sc-10848, lot \# G1806), RRM2b (N-16, cat \# sc10840, lot \# E2107), P38 (H-147, cat \# sc-7149, lot \# I149), ERCC1 (FL-297, cat \# sc-10785, lot \# G1103), and MCM2 (N-19, cat \# sc-9839, lot \# I1907) were purchased from Santa Cruz Biotechnology, and monoclonal antibody to TS (TS106, cat \# MS-471-p1, lot \# 471P708B) was from Anatomical Pathology and to TP53 (p53, cat \# 554293, lot \# 0000045190) from BD Bioscience. The bound antibody was detected using the ECL detection system according to manufacturer's instructions (Amersham Pharmacia Biotech). The intensity values of specific bands were quantified with a Personal Densitometer SI (Molecular Dynamics). To compare expression values among the different cell lines, the target protein values were normalized by comparison with the house keep gene $\beta$ actin. These adjusted measures were then assigned the value 1.0 in cell line $\mathrm{H} 23$ to obtain relative adjusted values for all other cell lines.

2.7. DNA Sequencing. DNA sequencing of the p53 and Kras genes was done using the Applied Biosystems 3130XL genetic analysis system. Genomic DNA from tumor cell lines was obtained with PureLink Genomic DNA kits (Invitrogen). Exons of the p53 gene were amplified using previously reported primers with minor modifications [11]. For K-ras, codon 12 and 13 were sequenced bidirectionally. For p53, all 11 exons were sequenced in both directions. All sequence data were confirmed with publicly available information.

\section{Results}

3.1. Impact of RRM1 Modulation on Different Classes of Agents. We had previously described the stably transfected RRM1 up- and down-regulated clones of NSCLC cell line H23 [4]. To expand and complement this model, we generated similar clones for the human breast cancer cell line MCF7 and colon cancer cell line HCT8. RRM1 expression at the mRNA and protein level was variable among clones. For drug testing, clones with a greater than 2-fold increase (for up regulation) or a greater than 2 -fold decrease (expression
$<50 \%$ of control) in RRM1 expression at the mRNA and protein level were selected (Figures 1(a), 1(b)).

To evaluate the impact of RRM1 on different classes of chemotherapeutic agents, clones with high and low RRM1 levels and their respective controls were treated with each agent over a broad range of concentrations. Dose response blots were generated and mean $\mathrm{IC}_{50}$ values calculated from at least 3 independent experiments (Table 1). For all agents, a dose-dependent inhibition was observed (Figure 1(c)). The relative impact of RRM1 was assessed by dividing the $\mathrm{IC}_{50}$ values of RRM1 modulated clones with those of control clones (Table 1). High RRM1 levels resulted in resistance and low levels in sensitivity to gemcitabine and 5-FU in NSCLC cell line H23, breast cancer cell line MCF7, and colon cancer cell line HCT8. Similarly, high RRM1 induced resistance to methotrexate and pemetrexed in $\mathrm{H} 23$, but low levels induced only a minimal increase in sensitivity. In MCF7 and HCT8, no effect on methotrexate was observed and the effect on pemetrexed was in the opposite direction; that is, high RRM1 was associated with increased sensitivity and low levels with resistance. Hydroxyurea was not affected by RRM1 in H23, but low levels resulted in increased sensitivity in MCF and HCT8.

For the platinum agents cisplatin and carboplatin, high or low RRM1 induced minimal resistance or sensitivity in H23 and had no consistent impact in MCF7 and HCT8. There was no observable relationship between RRM1 levels and efficacy of docetaxel, vinorelbine, and etoposide in all three model systems.

3.2. Impact of RRM1 Modulation on Drug Combinations in H23. We next assessed if RRM1 modulation would impact on the cytotoxicity of combinations of two agents. For this, we chose four commonly used chemotherapy doublets focused on antimetabolites in NSCLC; that is, gemcitabine + carboplatin, gemcitabine + docetaxel, gemcitabine + pemetrexed, and pemetrexed + carboplatin. The assays and analyses were as described using synchronous drug exposure, and a combination index (CI) was calculated from three separate experiments (Table 2). We observed synergy for the two platinum combinations and antagonism for the two nonplatinum combinations. RRM1 expression levels did not abrogate or reverse these interactions, although the CI values differed slightly among the RRM1 modulated clones.

3.3. Down Regulation of RRM1 by RNA Interference Increases Gemcitabine Sensitivity in Other NSCLC Cell Lines. To confirm if RRM1 downregulation would increase gemcitabine efficacy in other NSCLC cell lines, we transfected $20 \mathrm{nM}$ of target-specific short interfering RNA (siRNA) and nonspecific random siRNA for control purposes into cell lines A549, H292, and H460. Since ERCC1 (excision repair cross complementing group 1) expression levels in lung cancers are positively correlated with those of RRM1, we also used ERCC1-specific siRNA as a control. Immunoblot analysis demonstrated efficient knock-down of the specific target proteins RRM1 and ERCC1 in all three cell lines 


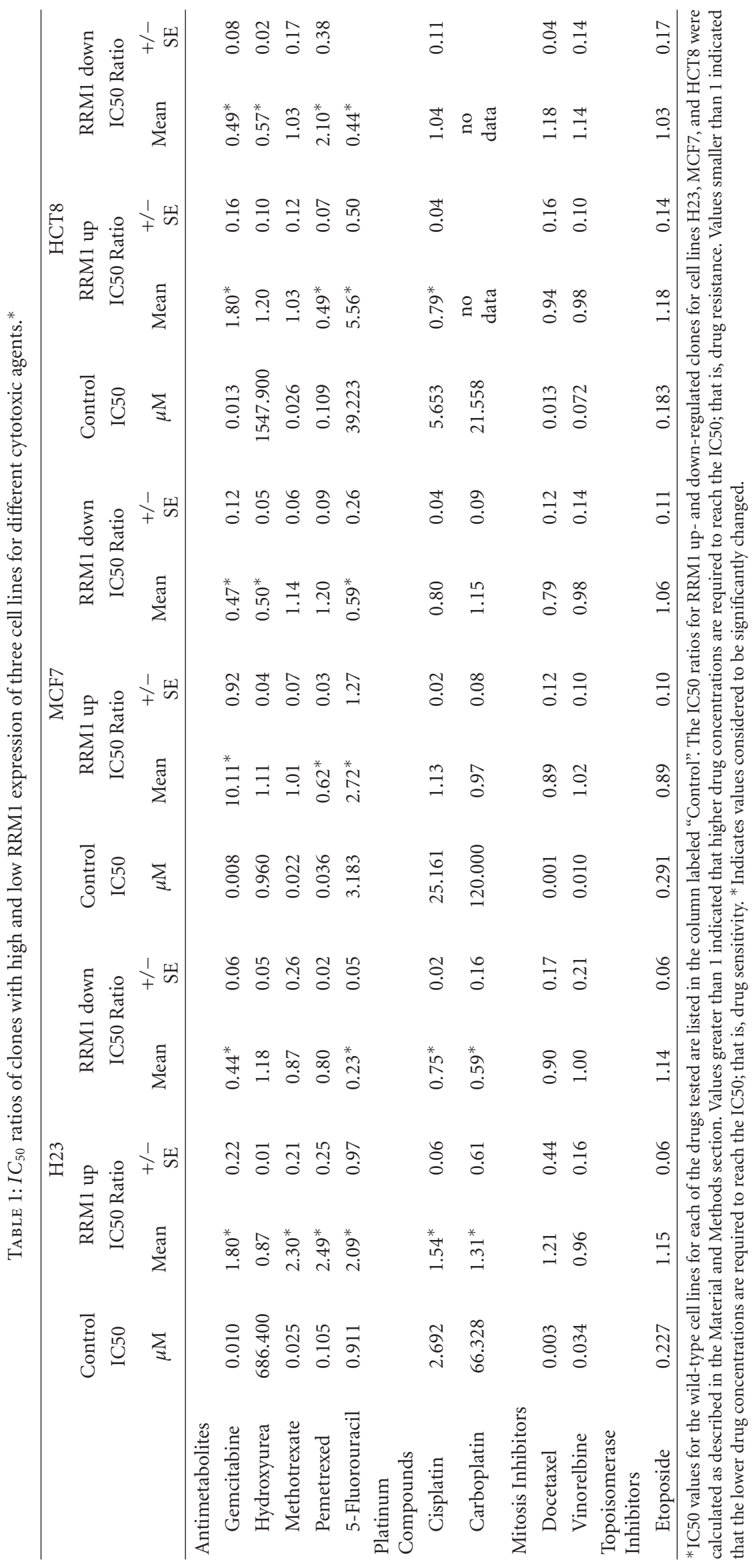




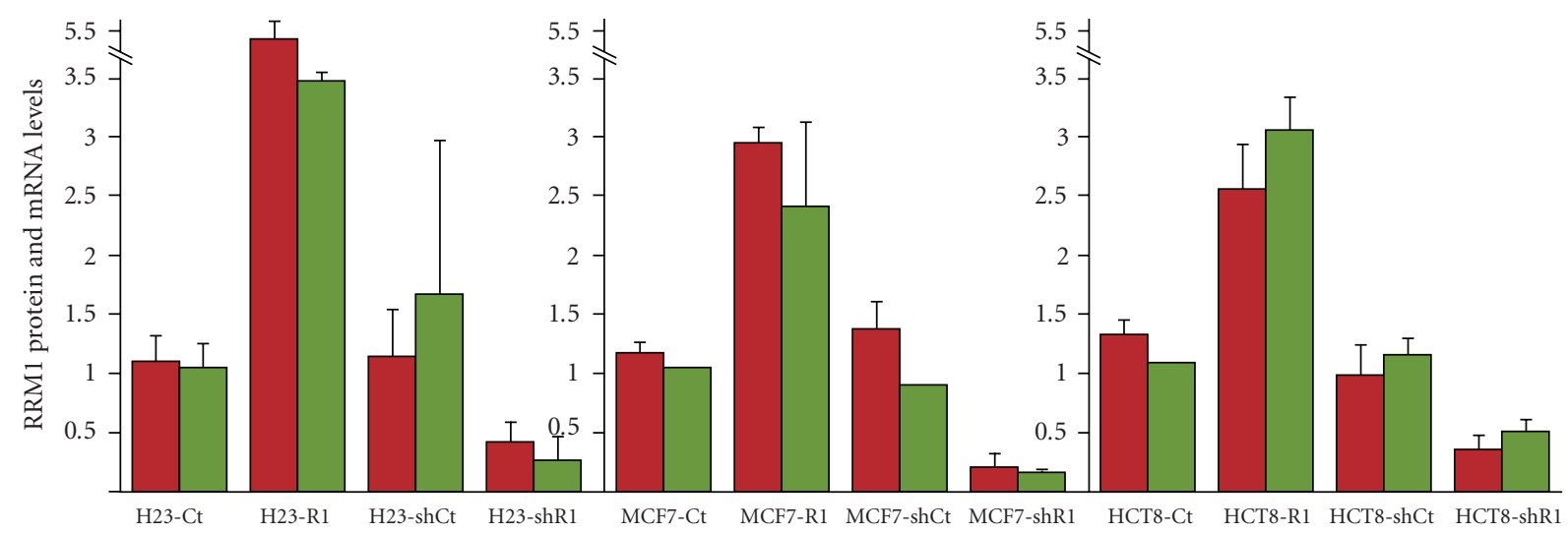

(a)

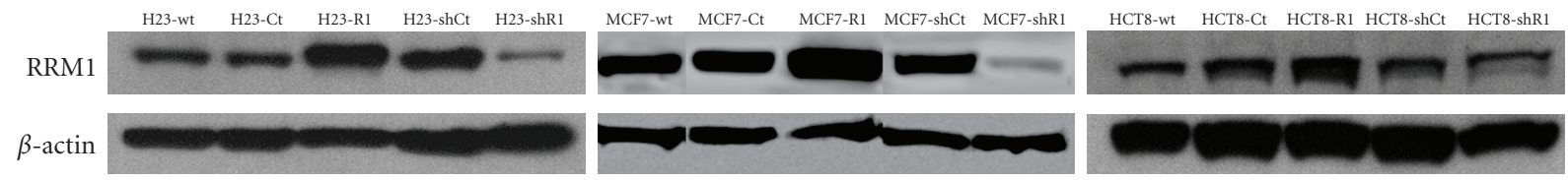

(b)

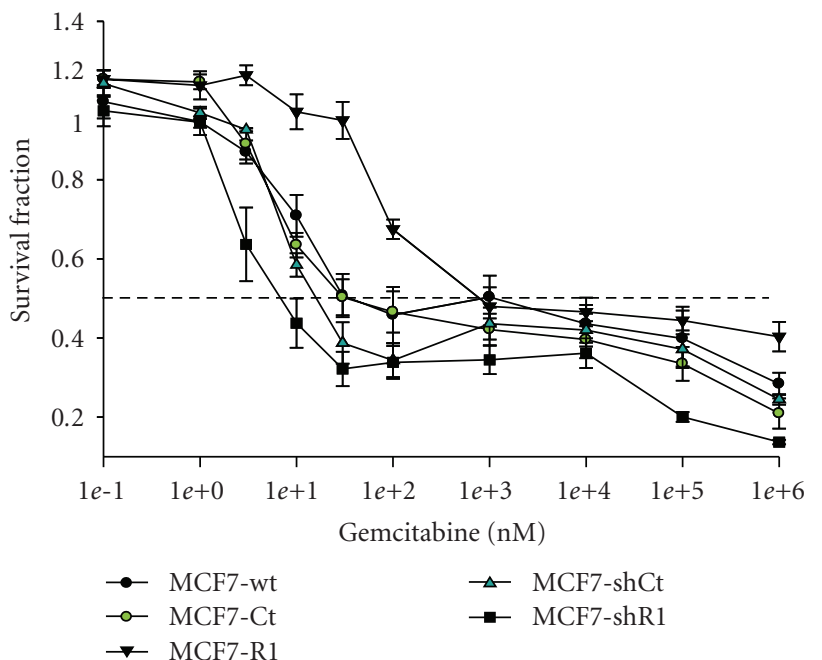

(c)

FIGURE 1: Modification of RRM1 expression by stable transfection with RRM1 and shRRM1 expression vectors in cell lines H23, MCF7, and HCT. Wt, wild-type cell lines, R1, clones of cell lines transfected with RRM1; Ct, clones transfected with an out-of-frame RRM1 vector; shR1, clones transfected with a small hair-pin RRM1 vector; shCt, clones transfected with a random control small hair-pin vector. (a) RRM1 protein (red) and mRNA (green) expression in stably transfected clones of H23, MCF7, and HCT8. (b) Western blots of H23, MCF7, and HCT8 clones. (c) Cytotoxicity of MCF7 clones following gemcitabine treatment for 6 days. Each point is the mean of at least three independent experiments. The dashed line indicates the $50 \%$ survival fraction.

(Figure 2(a)). We observed a 5- to 20-fold increase in gemcitabine efficacy with RRM1 down-regulation (Figure 2(b)); carboplatin efficacy was not notably affected.

\subsection{Endogenous RRM1 Expression, Drug Sensitivity, and Cell} Line Characteristics in a Panel of NSCLC Cell Lines. Since tumoral RRM1 levels and therapeutic efficacy of chemotherapy vary widely [7], we sought to investigate parameters that might influence the RRM1-gemcitabine efficacy interaction. For this, we used a random series of 26 NSCLC cell lines with a diverse range of properties. In these cell lines, we determined the endogenous levels of RRM1, RRM2a, RRM2b, and other molecules associated with nucleotide metabolism and cell proliferation in exponentially growing, subconfluent, and unsynchronized cultures. The relative expression levels were determined by densitometry of specific bands on a single, large immunoblot adjusted for $\beta$ actin expression and normalized to the level of each target protein in cell line $\mathrm{H} 23$ (levels arbitrarily set to 1.00). We also determined the p53 and K-ras mutational status, 
TABLE 2: Impact of RRM1 expression on drug combinations. *

\begin{tabular}{|c|c|c|c|c|}
\hline \multirow{3}{*}{ Drug combination } & \multicolumn{4}{|c|}{ Clones of $\mathrm{H} 23$} \\
\hline & $\mathrm{H} 23-\mathrm{Ct}$ & $\mathrm{H} 23-\mathrm{R} 1$ & $\mathrm{H} 23-\mathrm{shCt}$ & H23-shR1 \\
\hline & control & $\mathrm{R} 1$ increased & control & R1 decreased \\
\hline Gemcitabine \& Carboplatin & $0.93(+/-)$ & $0.83(++)$ & $0.56(+++)$ & $0.72(++)$ \\
\hline Gemcitabine \& Docetaxel & $2.8(---)$ & $2.1(---)$ & $3.1(---)$ & $1.4(--)$ \\
\hline Gemcitabine \& Pemetrexed & $1.5(---)$ & $1.4(--)$ & $1.7(---)$ & $1.7(---)$ \\
\hline Pemetrexed \& Carboplatin & $0.79(++)$ & $0.94(+/-)$ & $0.73(++)$ & $0.64(+++)$ \\
\hline
\end{tabular}

the doubling time (calculated with CurveExpert software), and the $\mathrm{IC}_{50}$ and maximum achievable cytotoxicity with gemcitabine, pemetrexed, and carboplatin (Table 3).

We did not observe a statistically significant correlation between RRM1 levels and the gemcitabine $\mathrm{IC}_{50}$ values (Spearman rank correlation coefficient $r=0.10, P=.65$ ). There was also no significant correlation between RRM1 levels and those of the other 7 proteins analyzed or the doubling time. Of note, TS levels and pemetrexed $\mathrm{IC}_{50}$ values were not correlated $(r=0.003, P=.99)$, neither were ERCC1 levels and carboplatin $\mathrm{IC}_{50}$ values $(r=0.07, P=$ $.75)$.

However, the median $\mathrm{IC}_{50}$ values for gemcitabine were approximately 10-fold higher in the group of 8 cell lines with p53 truncations, deletions, or null status $(0.3 \mu \mathrm{M})$ compared to the 18 cell lines without such mutations $(0.03 \mu \mathrm{M} ; P=.06$ by rank sum test). A similar trend was not observed for pemetrexed or carboplatin. The K-ras mutation status did not impact efficacy of the three agents tested.

The doubling time of cell lines was significantly correlated with gemcitabine $\mathrm{IC}_{50}$ values; that is, cell lines with long doubling times had higher $\mathrm{IC}_{50}$ values (Spearman rank correlation coefficient $r=0.63, P<.001$ ), and it was not correlated with the $\mathrm{IC}_{50}$ values of pemetrexed or carboplatin.

3.5. TP53 Levels and Gemcitabine Cytotoxicity. In order to study if wild-type p53 contributes to gemcitabine efficacy, we delivered p53-specific siRNA and nonspecific random siRNA to cell lines H23, A549, H292, and H460. We obtained near complete knock down in A549 and H292, a partial reduction in $\mathrm{H} 460$, and a minimal reduction in $\mathrm{H} 23$ using $20 \mathrm{nM}$ siRNA concentrations and $24 \mathrm{hrs}$ of exposure (Figure 3 ). Higher siRNA concentrations and longer exposure times did not yield better p53 reduction in $\mathrm{H} 23$ and $\mathrm{H} 460$. Gemcitabine $\mathrm{IC}_{50}$ values increased 2.0-fold in A549 (p53 wild-type) to 3.4-fold in H292 (p53 wild-type) and remained essentially unchanged in H23 (1.3-fold, p53 M246I missense) and H460 (1.1-fold, p53 wild-type).

To corroborate this result, we used the p53-null cell line H358 and its stably transfected and wild-type p53-expressing counterpart H358p53+ (Figure 4). We observed a statistically significant reduction in the gemcitabine $\mathrm{IC}_{50}$ from $15.3 \mathrm{nM}$ in $\mathrm{H} 358$ to $10.7 \mathrm{nM}$ in $\mathrm{H} 358 \mathrm{p} 53^{+}(P=.03$ by $t$-test; values are means of three independent experiments using 5 days of exposure), while the $\mathrm{IC}_{50}$ values for pemetrexed and carboplatin were not significantly different between these cell lines.

\section{Discussion}

The use of unselected double-agent chemotherapy has resulted in an approximately 50\% improvement in overall median survival of patients with advanced NSCLC [12]. The only criteria currently used for selection of agents are histology [13], toxicity profiles, and convenience of delivery. Two recent prospective clinical trials have demonstrated the feasibility of selecting individualized chemotherapy based on RRM1 and/or ERCC1 expression levels in tumor biopsy specimens $[14,15]$. Both trials also reported favorable response rates for patients receiving molecularly-based selected compared to unselected therapy. In two additional prospective trials in patients with metastatic stage III or stage IV disease, a statistically significant association between the tumoral expression levels of RRM1 and the magnitude of change in tumor burden with gemcitabine single-agent or gemcitabine and carboplatin double-agent therapy have been reported; that is, the lower the levels the better the response $[4,7]$.

The antitumoral activity of gemcitabine is a result of at least two separate actions. One is a presumed direct interaction with RRM1, with a resulting reduction of ribonucleotide reductase function and deoxynucleotide levels, and the other is incorporation into newly synthesized DNA, with a resulting chain termination. It is the presumed interaction with RRM1 that explains the direct and linear association between RRM1 levels and gemcitabine $\mathrm{IC}_{50}$ levels in experimental model systems. However, as can be gleaned from the published scatter plots depicting the association between intratumoral RRM1 levels and tumor response in cancer patients $[4,7]$, it is difficult to be precise in predicting whether an individual patient will actually derive benefit from the selected therapy. 


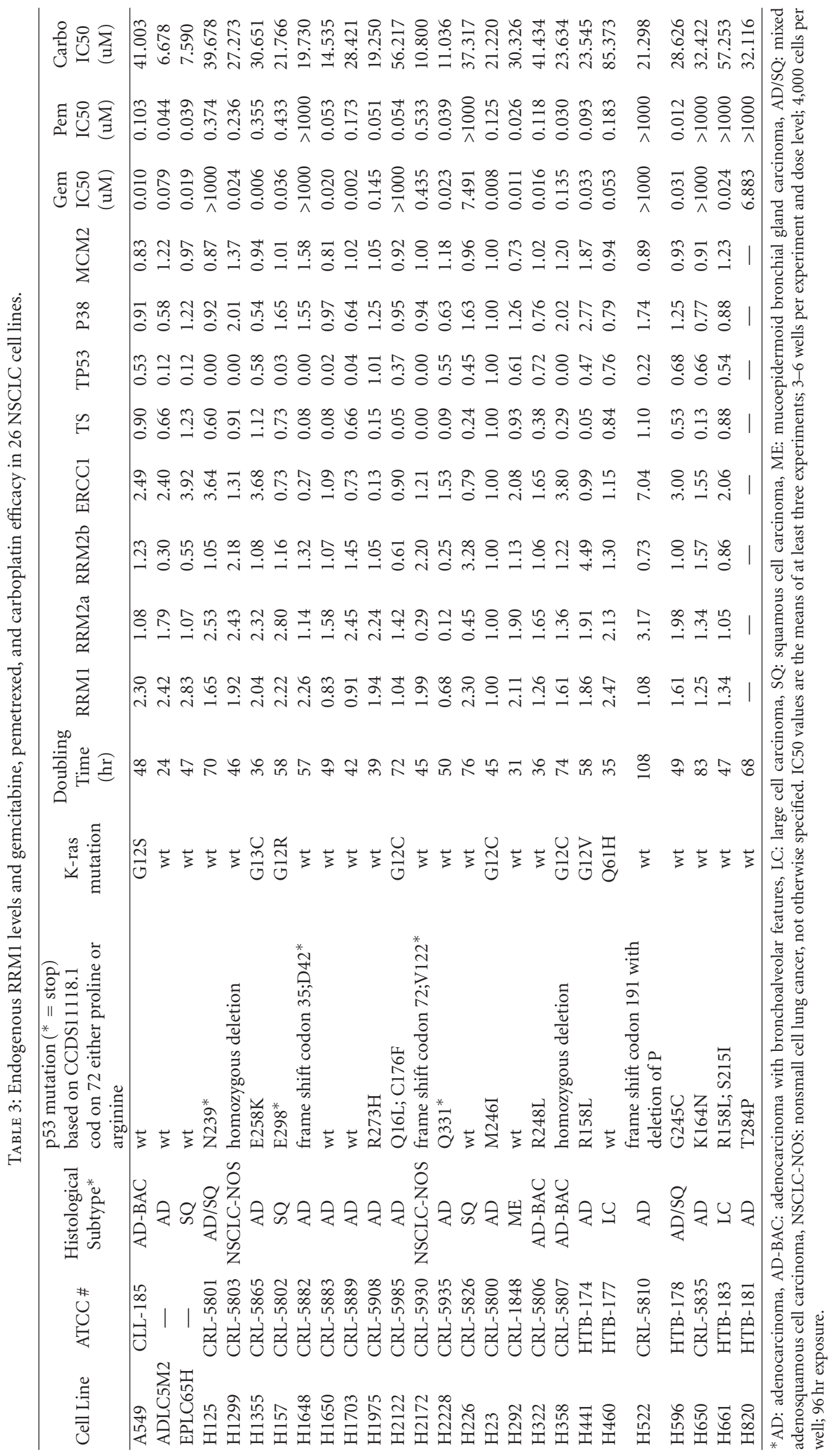



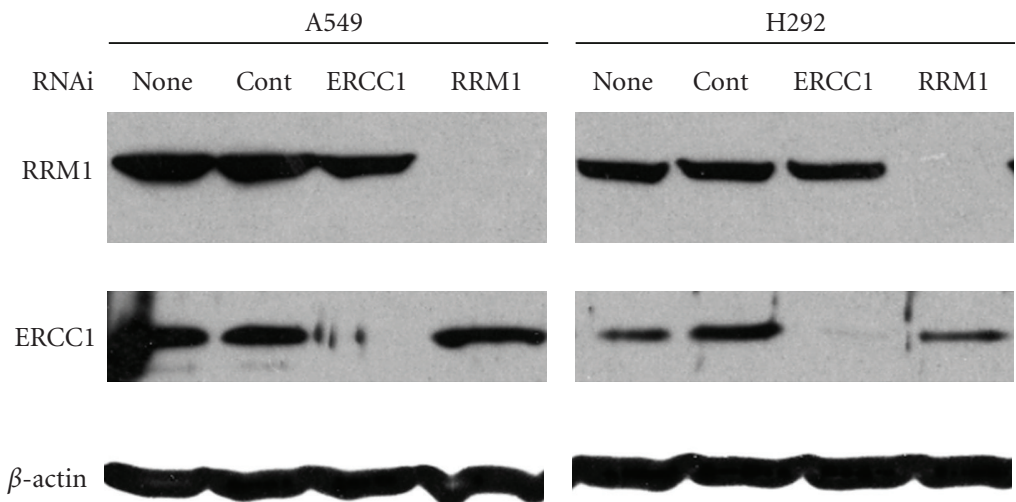

(a)
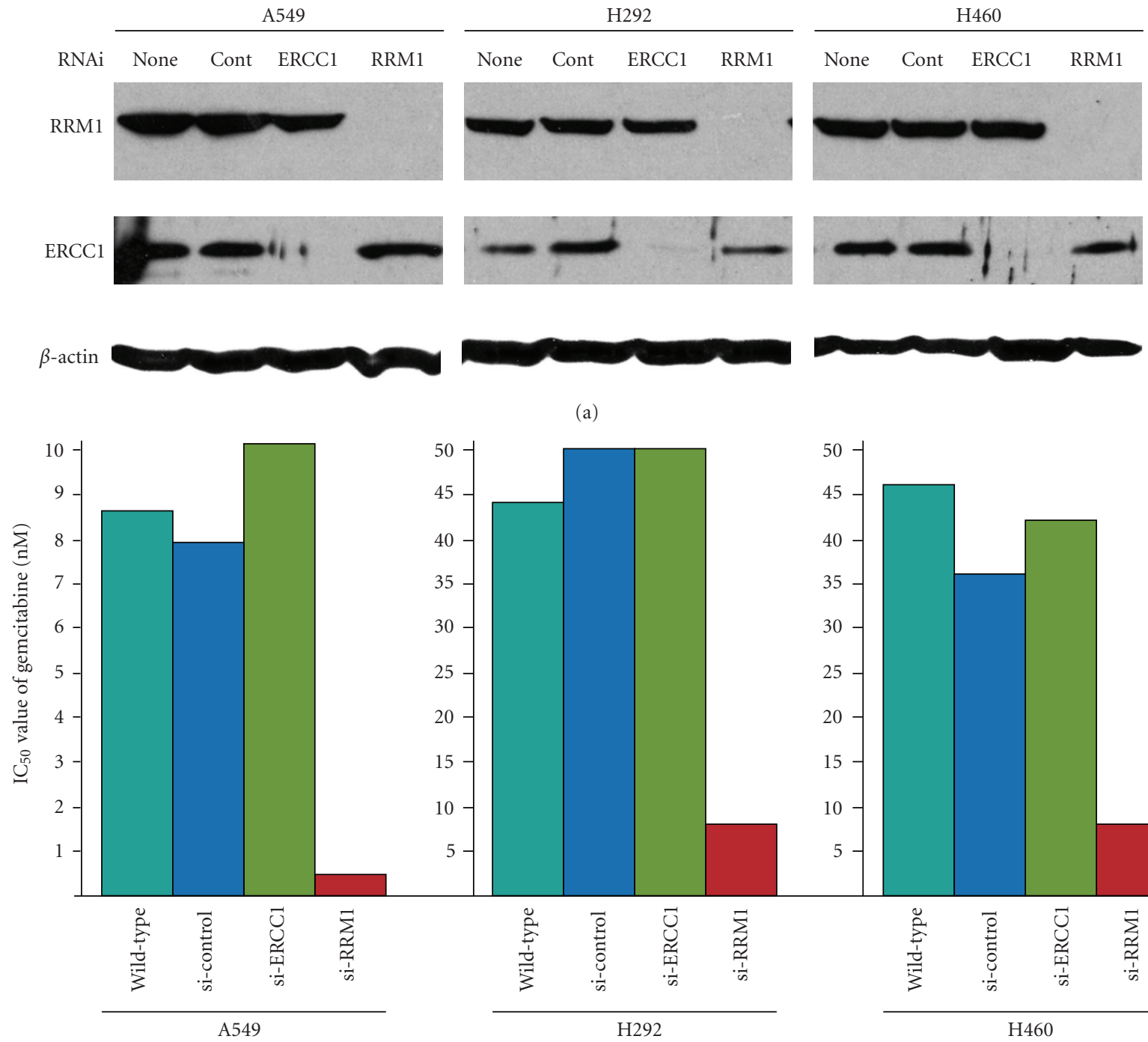

(b)

FIGURE 2: Knock-down of RRM1 and ERCC1 expression in three NSCLC cell lines by RNA interference and impact on gemcitabine efficacy. (a) Western blots showing that RRM1-specific siRNA reduced RRM1 protein expression to undetectable levels, while random control and ERCC1-specific siRNAs did not affect RRM1 expression. Likewise, ERCC1-specific siRNA reduced ERCC1 protein expression to undetectable levels, while random control and RRM1-specific siRNAs did not affect ERCC1 expression. (b) IC50 values of gemcitabine cytotoxicity in cell lines A549, H292, and H460. Wt, wild-type cell lines; si-control, cell lines transfected with nonspecific siRNA; si-ERCC1; cell lines transfected with ERCC1-specific siRNA; si-RRM1, cell lines transfected with RRM1-specific siRNA.

Given the molecular complexity of NSCLC, this is not surprising and strongly suggests that a variety of other tumor-specific and host-specific parameters substantially impact the gemcitabine-RRM1 interaction. Our results in a lung, breast, and colon cancer cell line with genetically modified RRM1 levels demonstrate that RRM1 expression levels are the dominant determinant of gemcitabine efficacy despite diverse molecular backgrounds. This result is consistent with prior reports of increased RRM1 levels in pancreatic and colon cancer models upon induction of gemcitabine resistance $[3,5]$. In our cell line models, we further demonstrate that RRM1 levels can impact efficacy of other cytotoxic agents in the class of antimetabolites. Since this effect was not observed in all cell lines, other parameters may dominate over the interaction between RRM1 and $5 \mathrm{FU}$, pemetrexed, and methotrexate. For instance, a significant role for TS on 5FU and dihydrofolate reductase on methotrexate efficacy has been established, and a role for TS and other enzymes involved in nucleotide synthesis on pemetrexed efficacy is evolving. However, an explanation for the increased pemetrexed sensitivity of RRM1 transfected MCF7 and HCT8 cell lines is elusive. We also demonstrate that RRM1 levels do not impact on efficacy of spindledisrupting agents or etoposide, which are frequently used in lung cancer therapy. In fact, an earlier report on a small subset of patients treated with vinorelbine, gemcitabine, and platinum had suggested that the addition of vinorelbine may abrogate the therapeutic benefit of gemcitabine in patients 

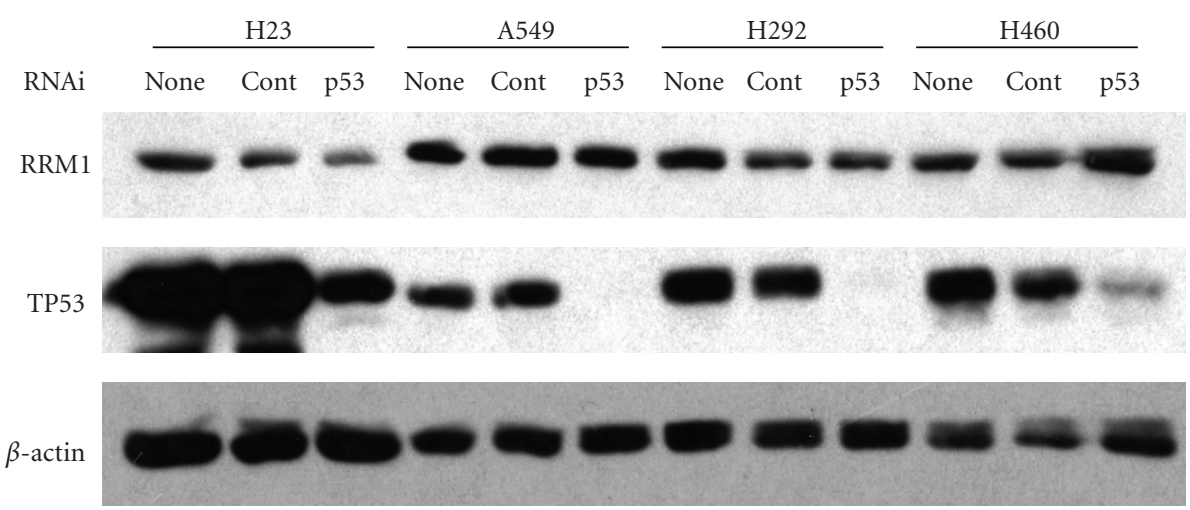

FIGURE 3: Western blots of knock-down of TP53 expression in four NSCLC cell lines by RNA interference. TP53-specific siRNA reduced TP53 protein expression to undetectable levels in A549 and H292 and greater then 10-fold in H23 and H460, while random control siRNA did not affect TP53 or RRM1 expression.

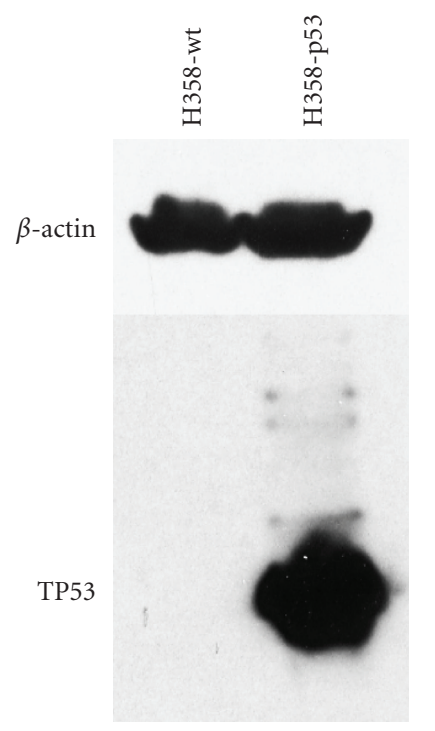

Figure 4: Western blots of cell line H358. There is no detectable TP53 expression in wild-type cells; while transfected cells clearly show TP53 expression.

with low levels of RRM1 expression [2]. Our in vitro results on combination therapy suggest that RRM1 does not disrupt drug-drug interactions when gemcitabine is combined with carboplatin, docetaxel, or pemetrexed.

Since RRM1 is often combined with ERCC1 in trials seeking to enhance therapeutic efficiency through agent selection and because both molecules are frequently coexpressed $[2,4,7,16,17]$, we tested in three cell lines if ERCC1 expression reduction through RNA interference would alter gemcitabine efficacy and found no evidence for this.

Using a panel of lung cancer cell lines with diverse features and molecular characteristics, we identified two variables that significantly impacted gemcitabine efficacy without being associated with RRM1 levels. We found that cell lines with functional p53-impairing mutations, that is, null, truncation, and deletion mutations, displayed a pattern of resistance to gemcitabine. We corroborated this result through transfection of wild-type p53 into a null cell line (H358), which resulted in a statistically significant improvement in gemcitabine efficacy, and through abrogation of p53 by RNA interference in other cell lines, which resulted in gemcitabine resistance. This was not explained by RRM1 expression levels, since no association between RRM1 levels and p53 was observed. In contrast, pemetrexed and platinum efficacy did not seem to be influenced by p53 expression modulation. To our knowledge, the impact of p53 mutations on gemcitabine efficacy has not been studied in clinical trials. It is important to corroborate these results in prospective trials since a potential clinical implication might be that tumoral RRM1 levels may not be predictive of gemcitabine efficacy in patients whose tumors harbor functionally significant p53 mutations.

Finally, our in vitro data demonstrated a statistically significant correlation between gemcitabine efficacy and the doubling time; that is, cell lines with long doubling times were more resistant to gemcitabine. Although we used a 4day continuous exposure to gemcitabine, which should allow for all cells to proceed through at least one complete cell cycle, we cannot exclude that this result is caused by the experimental conditions. However, a similar phenomenon was not observed for pemetrexed or carboplatin, which suggests that a true association between the speed of cellular replication and gemcitabine efficacy exists. It is not explained by RRM1 expression levels or p53, since a significant correlation between these and the doubling time was not observed. However, we had previously reported that overexpression of RRM1 through stable transfection in cell lines resulted in slow growth predominantly through G2 arrest [18]. It is thus possible that the increased gemcitabine efficacy is a result of a decreased ability of cells to repair newly synthesized DNA with incorporated $2^{\prime}, 2^{\prime}$-difluorodeoxycytidine that leads to chain termination [19]. 


\section{Conclusions}

We demonstrated a dominant role for RRM1 in gemcitabine efficacy and also a role in efficacy of other antimetabolites in selected cell lines. RRM1 did not disrupt the interaction between gemcitabine and other cytotoxic agents when combined. The mutational status of p53 and cell line doubling time were significant and independent determinants of gemcitabine efficacy. Their impact on gemcitabine efficacy in patients with lung cancer in the context of RRM1 expression requires investigations in prospective clinical trials.

\section{Abbreviations}

RR: $\quad$ Ribonucleotide reductase

RRM1: RR subunit M1

RRM2a: RR subunit M2a

RRM2b: p53-inducible RR subunit M2b, encoded by the p53R2 gene

ERCC1: Excision repair cross-complementation group 1

TP53: p53 tumor suppressor gene

TS: Thymidylate synthase

MCM2: Minichromosome maintenance protein 2

MTS: 3-(4,5-dimethylthiazol-2-yl)-5(3- carboxymethoxyphenyl)-2-

(4-sulfophenyl)-2H-tetrazolium

NSCLC: Nonsmall cell lung cancer.

\section{Acknowledgment}

This work was supported by R01-CA129343 from the NCI.

\section{References}

[1] J. D. Davidson, L. Ma, M. Flagella, S. Geeganage, L. M. Gelbert, and C. A. Slapak, "An increase in the expression of ribonucleotide reductase large subunit 1 is associated with gemcitabine resistance in non-small cell lung cancer cell lines," Cancer Research, vol. 64, no. 11, pp. 3761-3766, 2004.

[2] R. Rosell, K. D. Danenberg, V. Alberola et al., "Ribonucleotide reductase messenger RNA expression and survival in gemcitabine/cisplatin-treated advanced non-small cell lung cancer patients," Clinical Cancer Research, vol. 10, no. 4, pp. 1318-1325, 2004.

[3] A. M. Bergman, P. P. Eijk, V. W. T. Ruiz van Haperen et al., "In vivo induction of resistance to gemcitabine results in increased expression of ribonucleotide reductase subunit M1 as the major determinant," Cancer Research, vol. 65, no. 20, pp. 9510-9516, 2005.

[4] G. Bepler, I. Kusmartseva, S. Sharma et al., "RRM1 modulated in vitro and in vivo efficacy of gemcitabine and platinum in non-small-cell lung cancer," Journal of Clinical Oncology, vol. 24, no. 29, pp. 4731-4737, 2006.

[5] S. Nakahira, S. Nakamori, M. Tsujie et al., "Involvement of ribonucleotide reductase M1 subunit overexpression in gemcitabine resistance of human pancreatic cancer," International Journal of Cancer, vol. 120, no. 6, pp. 1355-1363, 2007.

[6] H. Akita, Z. Zheng, Y. Takeda et al., "Significance of RRM1 and ERCC1 expression in resectable pancreatic adenocarcinoma," Oncogene, vol. 28, no. 32, pp. 2903-2909, 2009.
[7] C. Reynolds, C. Obasaju, M. J. Schell et al., "Randomized phase III trial of gemcitabine-based chemotherapy with in situ RRM1 and ERCC1 protein levels for response prediction in non-small-cell lung cancer," Journal of Clinical Oncology, vol. 27, no. 34, pp. 5808-5815, 2009.

[8] G. Bepler, K. E. Sommers, A. Cantor et al., "Clinical efficacy and predictive molecular markers of neoadjuvant gemcitabine and pemetrexed in resectable non-small cell lung cancer," Journal of Thoracic Oncology, vol. 3, no. 10, pp. 1112-1118, 2008.

[9] A. Gautam, Z.-R. Li, and G. Bepler, "RRM1-induced metastasis suppression through PTEN-regulated pathways," Oncogene, vol. 22, no. 14, pp. 2135-2142, 2003.

[10] T.-C. Chou, "Theoretical basis, experimental design, and computerized simulation of synergism and antagonism in drug combination studies," Pharmacological Reviews, vol. 58, no. 3, pp. 621-681, 2006.

[11] M. Behn and M. Schuermann, "Sensitive detection of p53 gene mutations by a 'mutant enriched' PCR-SSCP technique," Nucleic Acids Research, vol. 26, no. 5, pp. 1356-1358, 1998.

[12] T. D. Shanafelt, C. Loprinzi, R. Marks, P. Novotny, and J. Sloan, "Are chemotherapy response rates related to treatmentinduced survival prolongations in patients with advanced cancer?" Journal of Clinical Oncology, vol. 22, no. 10, pp. 19661974, 2004.

[13] G. V. Scagliotti, P. Parikh, J. Von Pawel et al., "Phase III study comparing cisplatin plus gemcitabine with cisplatin plus pemetrexed in chemotherapy-naive patients with advancedstage non-small-cell lung cancer," Journal of Clinical Oncology, vol. 26, no. 21, pp. 3543-3551, 2008.

[14] G. Simon, A. Sharma, X. Li et al., "Feasibility and efficacy of molecular analysis-directed individualized therapy in advanced non-small-cell lung cancer," Journal of Clinical Oncology, vol. 25, no. 19, pp. 2741-2746, 2007.

[15] M. Cobo, D. Isla, B. Massuti et al., "Customizing cisplatin based on quantitative excision repair cross-complementing 1 mRNA expression: a phase III trial in non-small-cell lung cancer," Journal of Clinical Oncology, vol. 25, no. 19, pp. 27472754, 2007.

[16] P. Ceppi, M. Volante, S. Novello et al., "ERCC1 and RRM1 gene expressions but not EGFR are predictive of shorter survival in advanced non-small-cell lung cancer treated with cisplatin and gemcitabine," Annals of Oncology, vol. 17, no. 12, pp. 1818-1825, 2006.

[17] Z. Zheng, T. Chen, X. Li, E. Haura, A. Sharma, and G. Bepler, "DNA synthesis and repair genes RRM1 and ERCC1 in lung cancer," New England Journal of Medicine, vol. 356, no. 8, pp. 800-808, 2007.

[18] A. Gautam and G. Bepler, "Suppression of lung tumor formation by the regulatory subunit of ribonucleotide reductase," Cancer Research, vol. 66, no. 13, pp. 6497-6502, 2006.

[19] P. Huang, S. Chubb, L. W. Hertel, G. B. Grindey, and W. Plunkett, "Action of 2'2'-difluorodeoxycytidine on DNA synthesis," Cancer Research, vol. 51, no. 22, pp. 6110-6117, 1991. 

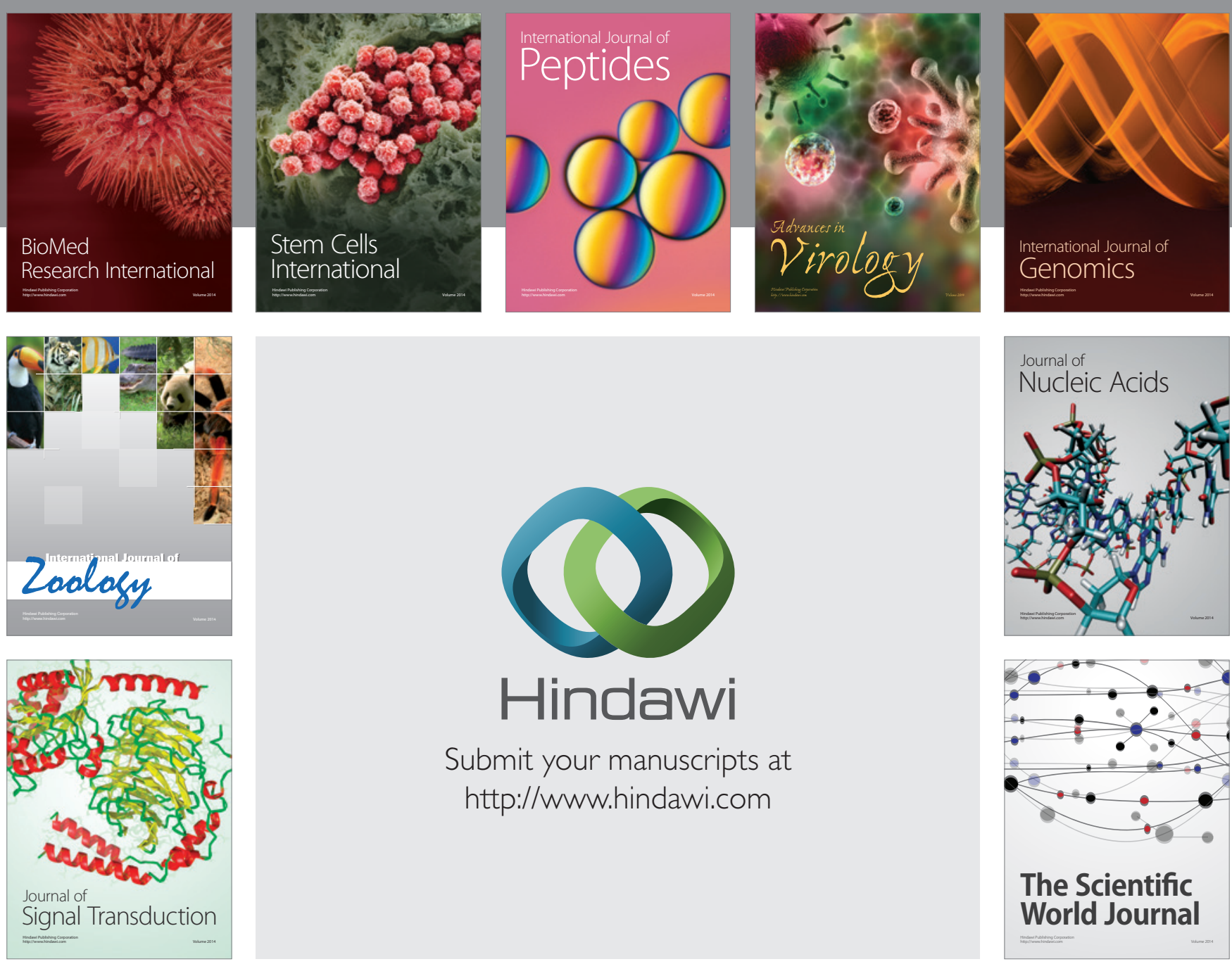

Submit your manuscripts at

http://www.hindawi.com
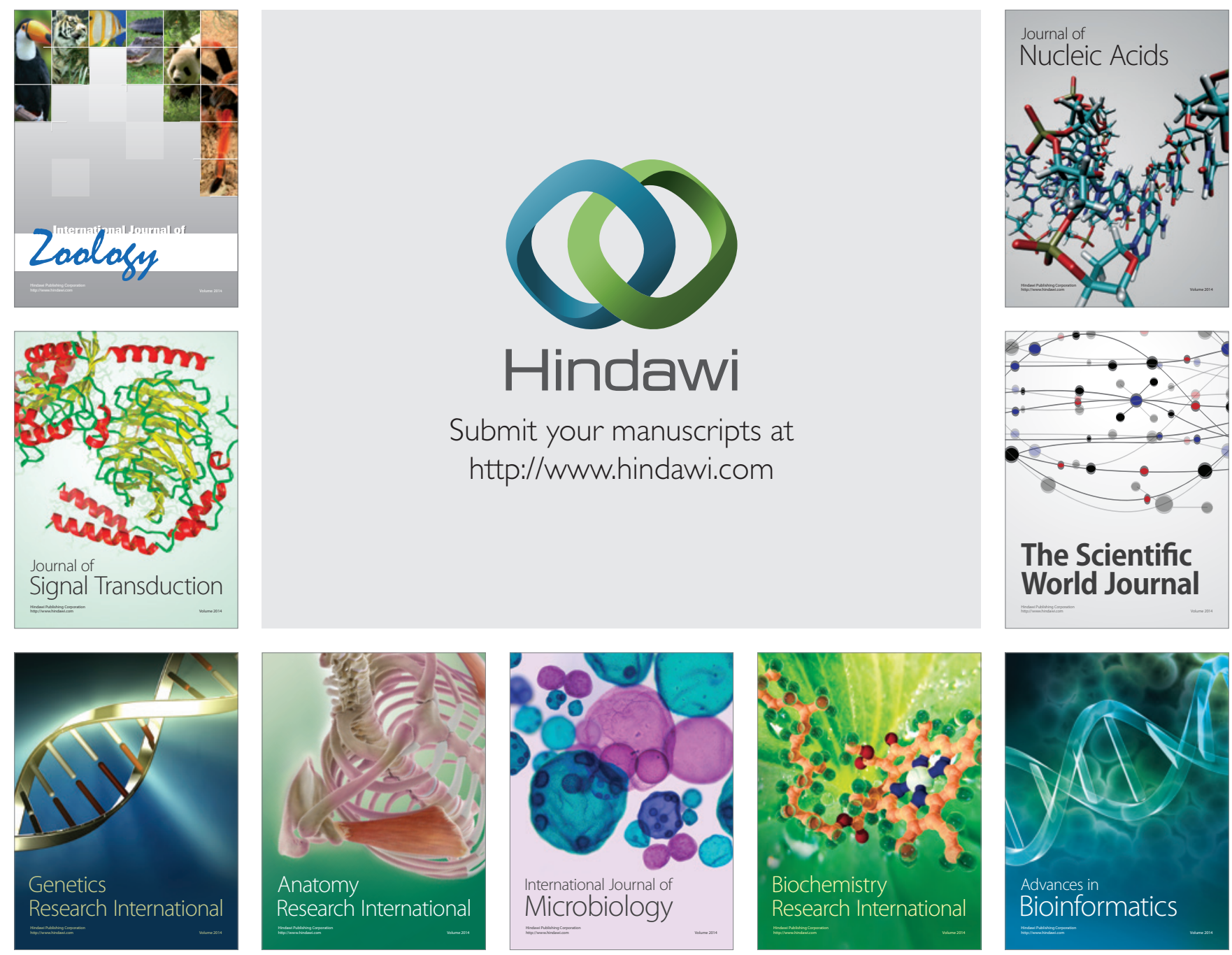

The Scientific World Journal
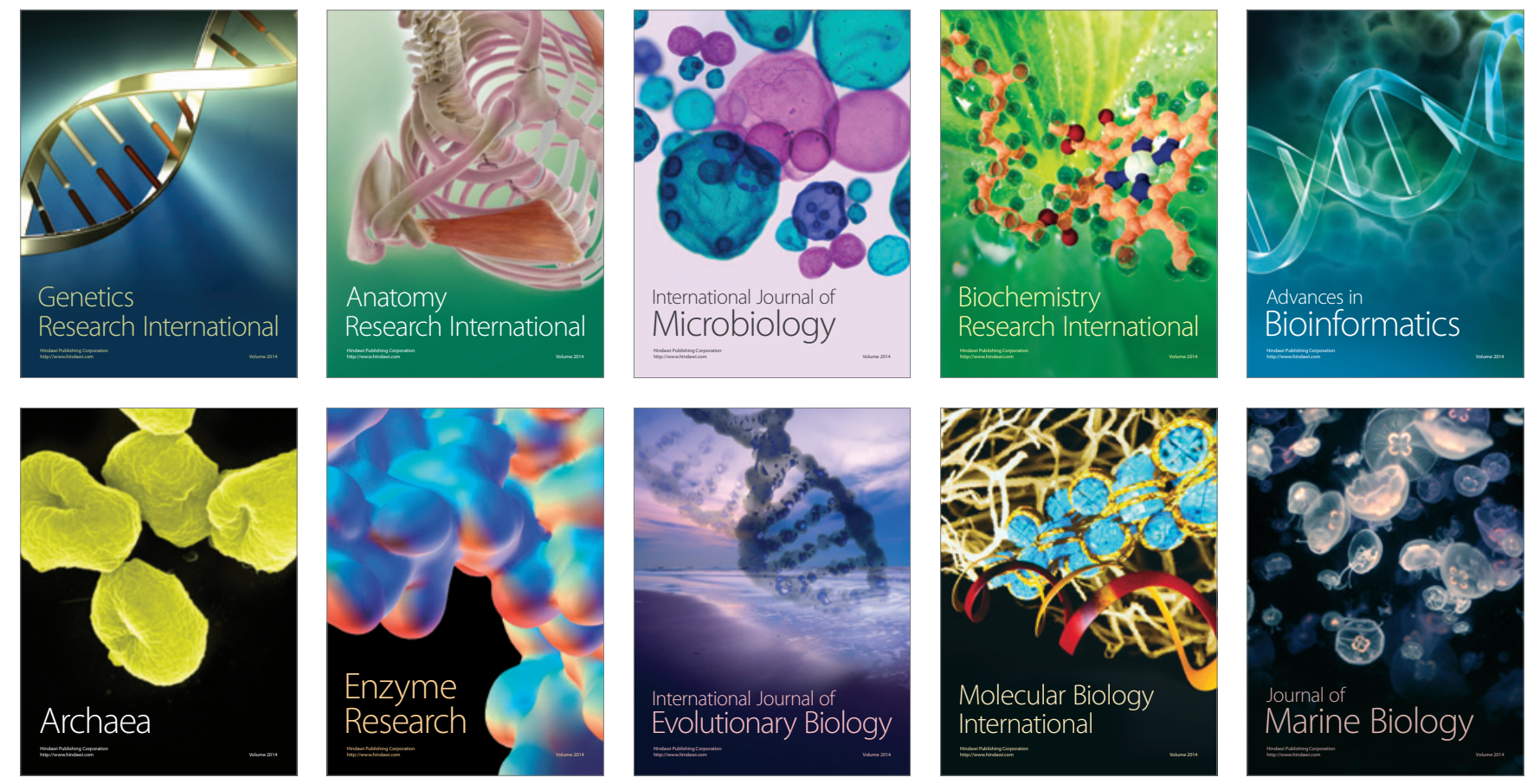